\title{
Multi-organ echinococcosis in a paediatric patient
}

\section{Juruan de Witt, MB ChB \\ Department of Radiology, Edendale Hospital, Pietermaritzburg \\ AF Stoker, $M B$ ChB \\ Department of Radiology, Grey's Hospital, Pietermaritzburg}

\section{Abstract}

Echinococcosis is an infection caused in humans by the larval stage of the Echinococcus parasite. Multi-organ involvement is common, and cysts may develop in various organs. Radiological imaging modalities play an important role in the diagnosis, management and follow-up of echinococcosis. We present a case of multi-organ echinococcosis with extensive pulmonary involvement.

\section{Introduction}

Echinococcosis (hydatid disease) is a potentially fatal disease that can affect animals and humans. Patients usually become symptomatic when larval cysts elicit space-occupying effects. By this stage, extensive multiorgan involvement may be present, as was demonstrated in this case.

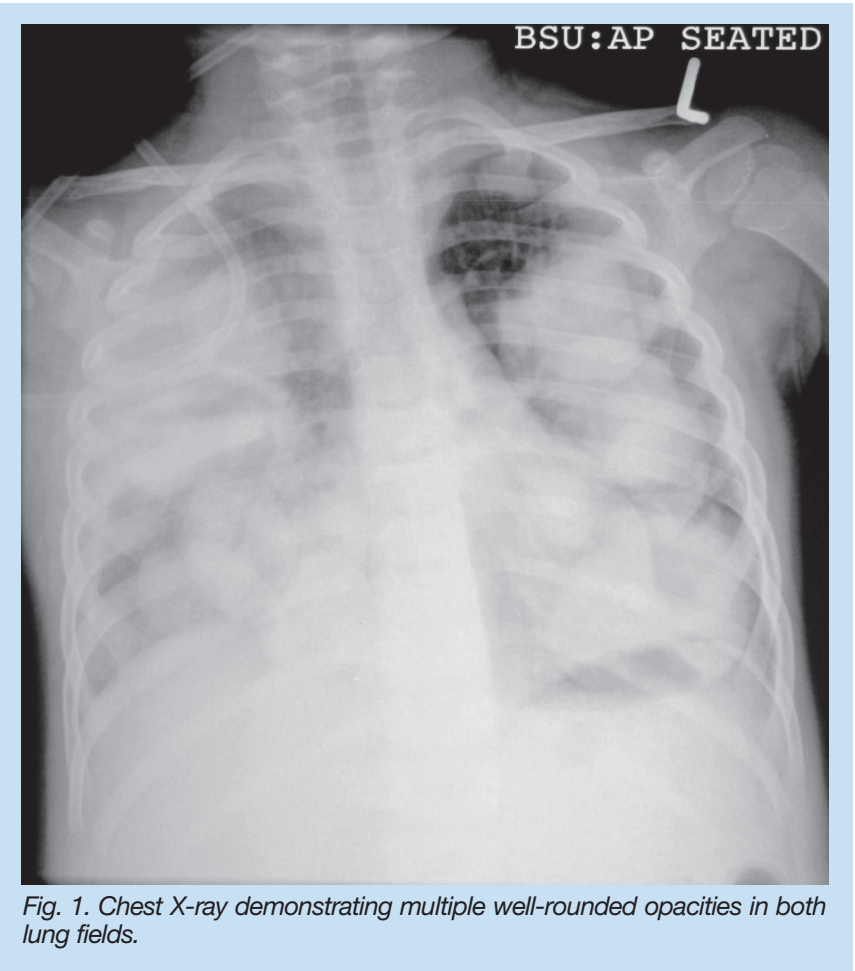

\section{Case report}

The 3-year-old patient presented with acute-onset respiratory distress and temperature spikes. The patient had had a chronic cough for 3

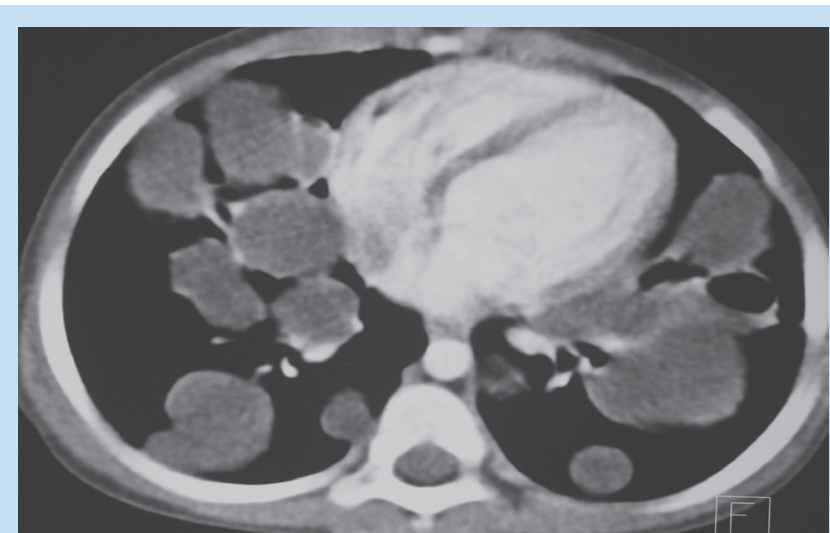

Fig. 2. Axial CT scan of the chest. The cystic nature of the pulmonary lesions can be appreciated.

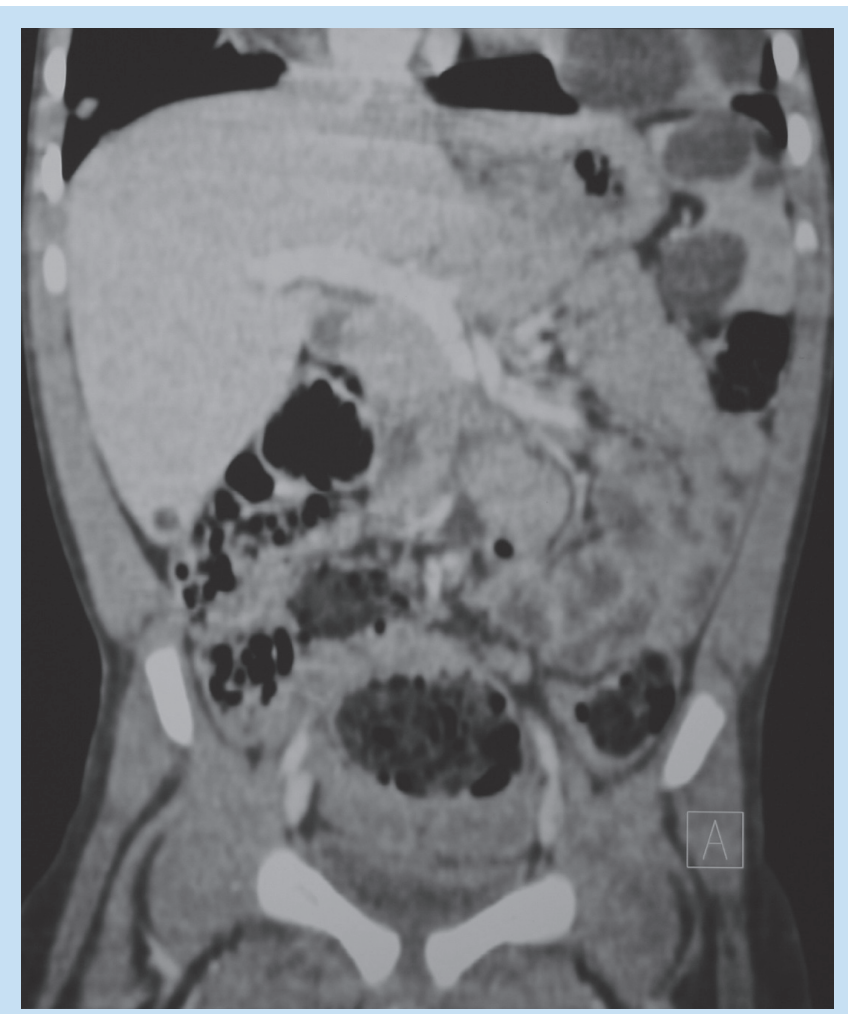

Fig. 3. Coronal CT scan of the abdomen. Three cysts noted in the spleen, as well as a solitary cyst in the inferior region of the liver.

months prior to this point. Auscultation of the lungs revealed bilateral coarse crackles, while the rest of the physical examination was unremarkable. A chest X-ray (CXR) showed bilateral well-defined rounded opacities involving all the lung zones (Fig. 1). A CT (computed tomography) scan of the chest revealed the cystic nature of these pulmonary lesions, while a CT scan of the abdomen demonstrated further cystic lesions in the spleen and the liver (Figs 2 and 3). The indirect haemagglutination (IHA) serological test was positive for hydatid disease. The 


\section{CASE REPORT}

patient was commenced on albendazole, prednisone and Berotec, and responded well. A follow-up CXR after 3 months' treatment showed marked improvement of the lesions (Fig. 4).

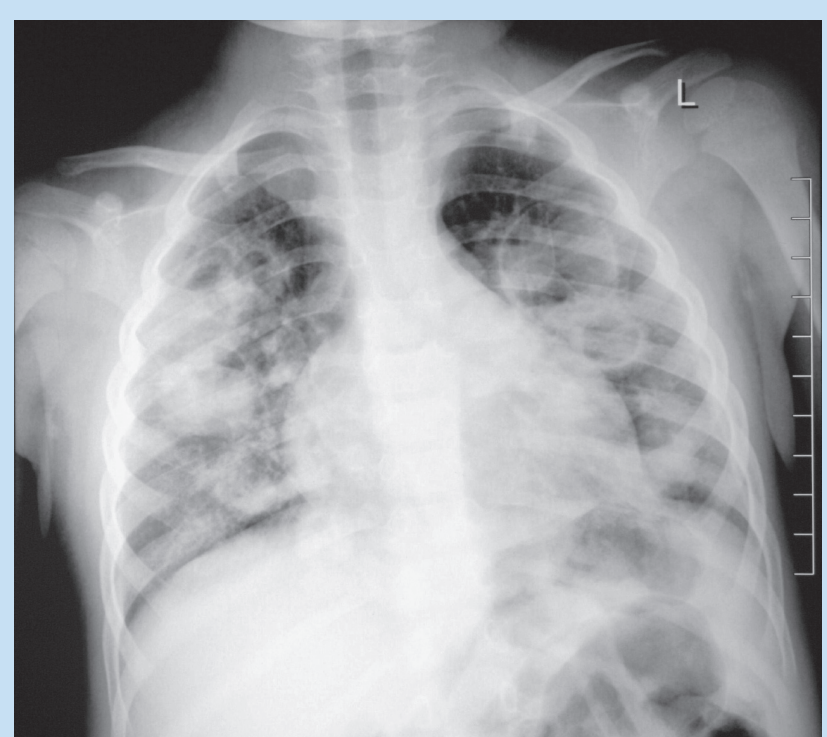

Fig. 4. Follow-up CXR after 3 months, demonstrating improvement of the pulmonary cysts.

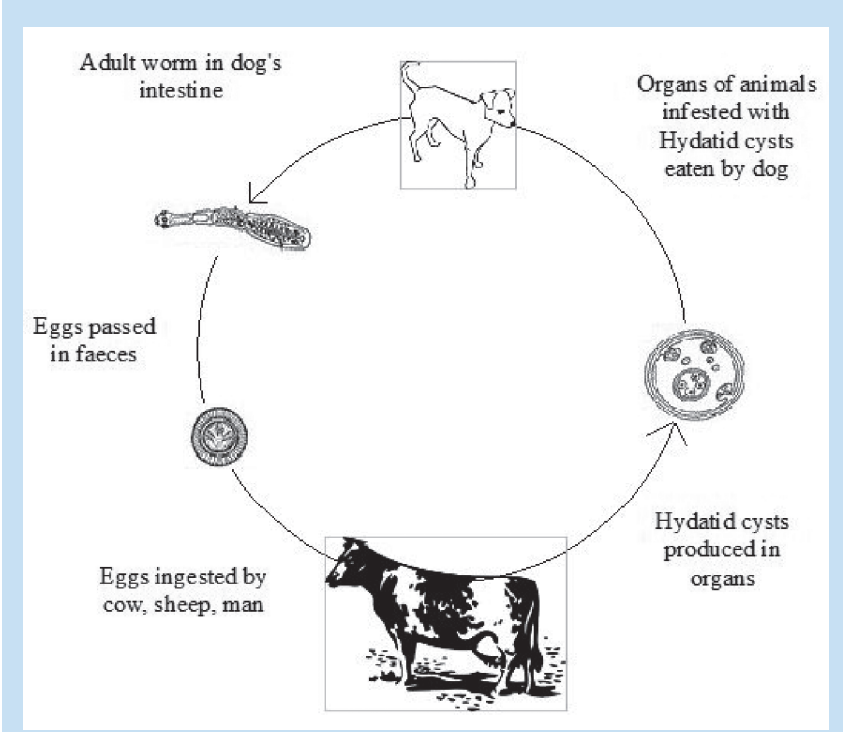

Fig. 5. The life cycle of E. granulosis. ${ }^{4}$

\section{Discussion}

Echinococcosis is an infection caused in humans by the larval stage of Echinococcus granulosis, E. multilocularis, or E. vogeli. E. granulosis produces unilocular cysts and is most prevalent in areas where livestock is raised in association with dogs. ${ }^{1}$

Humans are accidental hosts and are infected by the ingestion of ova from fomites or contaminated water, and by direct contact with dogs. After ingestion, embryos escape from the ova, penetrate the intestinal mucosa and enter the portal system, from where they are carried to various organs, most commonly the liver and the lungs. Here they develop

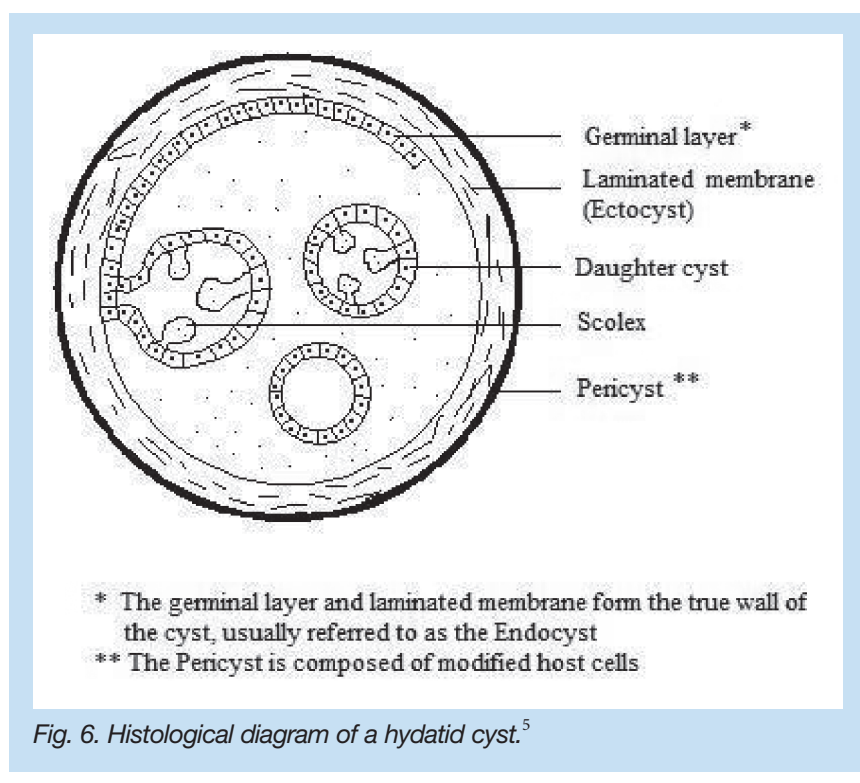

into mature larval cysts. ${ }^{2}$ Unless these slowly enlarging cysts elicit spaceoccupying effects on specific organs, an infected patient would usually remain asymptomatic. Pulmonary symptoms include cough, chest pain and haemoptysis, while hepatic involvement may cause fever, right upper quadrant pain, pruritis, urticaria, or eosinophilia. Other presentations may include anaphylaxis, pathological fractures, neurological deficit, pericarditis, arrhythmias and pelvic masses. ${ }^{2}$

Radiographic imaging studies are important in detecting and evaluating echinococcal cysts. Plain films will reveal unruptured pulmonary cysts as rounded masses of uniform density, while ruptured cysts result in complex cavitary lesions with variable radiographic features, which include an air-fluid level, a floating membrane (water-lily sign), a double wall, an essentially dry cyst with crumpled membranes (serpent sign, rising sun sign) or an empty cyst. ${ }^{2,3}$

Plain films may also be helpful in detecting cysts in other organs, e.g. calcified cysts in the liver. Ultrasound, CT and MRI may all reveal well-defined cysts with thick or thin walls. On CT, it is occasionally possible to demonstrate daughter cysts within a larger cyst; this has the characteristic appearance of eggshell or mural calcification and is virtually pathognomonic of E. granulosis infection, which is helpful when distinguishing echinococcus cysts from carcinomas, abscesses or haemangiomas. ${ }^{1}$

The indirect haemagglutination serological test is one of the most sensitive serological tests for the diagnosis of echinococcosis.

Treatment modalities include medical therapy with albendazole, percutaneous aspiration, infusion of scolicidal agents and re-aspiration of cyst content (PAIR) and surgical resection. Treatment is based on considerations of the size, location and manifestations of the cysts, as well as the overall health of the patient. The condition can be prevented by treating and vaccinating animals as well as encouraging basic human hygienic practices.

\section{Conclusion}

Although echinococcosis is mainly prevalent in rural areas, patients present at all levels of health care owing to complications of the disease or the lack of a definitive diagnosis. Various radiological modalities 


\title{
CASE REPORT
}

are used in the diagnosis, treatment and follow-up of echinococcosis Prompt recognition of the radiological features may prevent serious lifethreatening complications, which makes the radiologist an important role-player in the management of these patients.

1. Kasper DL, Braunwald E, Fauci AS, Hauser SL, Longo DL, Jameson JL. Harrison's Principles of Internal Medicine. 16th ed. New York: McGraw-Hill, 2005: 1275.
2. Grainger RG, Allison D, Adam A, Dixon AK. Diagnostic Radiology. 4th ed. New York: Churchill Livingstone, 2001: 400-401.

3. Dahnert W. Radiology Review Manual. 6th ed. Philadelphia: Lippincott Williams \& Wilkins, 2007: 500501.

4. Palmer PES, Reeder MM. The Imaging of Tropical Diseases: With Epidemiological, Pathological and Clinical Correlation. 2nd ed. Heidelberg, 2001: 206-222.

5. Pedrosa I, Saiz A, Arrazola J, Ferreirós J, Pedrosa CS. Hydatid disease: Radiologic and pathologic features and complications. RadioGraphics 2000; 20: 795-817.

\section{RSSA NEURORADIOLOGY AND BODY IMAGING ADVANCES CONGRESS}

\author{
28 - 30 AUGUST 2009 \\ SANDTON CONVENTION CENTRE
}
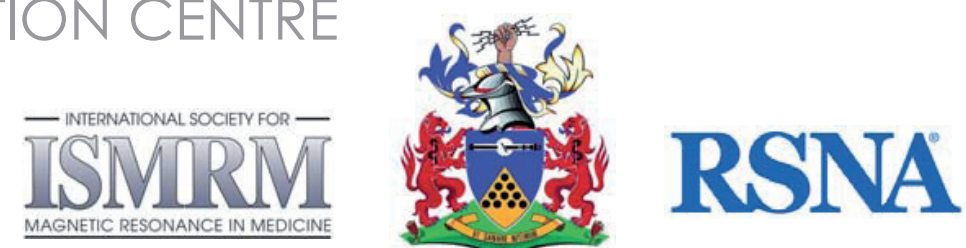

JOIN US AT THE FIRST JOINT MEETING BETWEEN:

- The Radiological Society of South Africa (RSSA)

- The Radiological Society of North America International Visiting Professors Programme (RSNA)

- The International Society for Magnetic Resonance in Medicine (ISMRM) Global Outreach Programme

For programme information and to register online, please visit our website at www.rssaneurorad2009.co.za 